\title{
Real-time interactive social environments: a review of BT's Generic Learning Platform
}

\author{
Michael Gardner and Holly Ward \\ Internet and Multimedia Application, BT Adastral Park, \\ email:michael.r.gardner@btcom
}

Online learning in particular and lifelong learning in general require a learning platform that makes sense both pedagogically and commercially. This paper sets out to describe what we mean by generic, learning and platform. The technical requirements are described, and various trials that test the technical, educational and commercial nature of the platform are described. Finally, the future developments planned for the Real-time Interactive Social Environments (RISE) are discussed.

\section{Introduction}

The vision of a single platform that supports all the key stakeholders within a wide range of distance learning activities has long been a dream of educationalists and commercial organizations alike. Such a platform could not only provide a consistent educational 'experience' for teachers and learners throughout their lives, but it could also provide administration and managerial support, all of which can be tailored to individual requirements.

This paper reviews the work carried out within BT's Education and Training Research Programme over the past three years in developing a generic platform for online distance learning. The first section of the paper reviews the original objectives of the Generic Learning Platform (GLP) and the context of the education and training research within BT. Following this, a description of the Real time Interactive Social Environment (RISE) platform is provided including the technical components of RISE.

Over the past three years the research team have carried out a number of trials using RISE. Each of these intended to stretch the overall architecture and our own understanding of what enables successful distance learning across a range of educational settings. An overview of each of these trials is given and the implications for implementing a GLP. Finally this paper closes with some comments on the future of RISE. 


\section{The meaning of generic}

In the context of our work, we define 'generic' as a solution that is not dependent upon any particular individual's learning stage, be it at school, college, university or work. Such a definition is essential to support meaningfully the notion of lifelong learning. The concept of learning being generic has two important implications. First it assumes that there is greater commonality rather than differences at the developmental stages of learning throughout most of our lives. The learning process is essentially universal, although it may require degrees of customization to meet some of the specific needs of the learners at their different developmental stages, situations or locations. Many of these learning issues will be addressed in the next section.

Generic solutions also make considerable commercial sense. Solutions specifically designed for each sector (i.e. schools, colleges, universities and learning for leisure) are expensive and may not facilitate movement between sectors as required by a lifelong learning scenario. $A$ generic solution recognizes a common core which may be customized to suit the needs of a sector (see section on the platform for more details on customization). Finally the term 'generic' is also making a statement about content that has both educational and commercial implications. Content is the critical component that needs to change to reflect the differing educational requirement - it cannot be generic across sector, at best it is common for a single homogeneous group of learners. The GLP, with respect to content at least, must be considered as a generic 'shell' into which appropriate content can be added. Commercially this also makes sense as BT does not consider itself as a content provider and works with third parties who have a high reputation in the area of content provision.

Our aim therefore was to produce a platform that was sufficiently generic to allow the 'reuse' of basic or core components across the market sectors, thus keeping development costs down, and sufficiently universal in terms of learning to provide a shell that requires the minimum of customization. To achieve this aim requires a conceptual framework that serves as a bridge between the theoretical academic educational research and the practicalities of implementing real distance learning systems. It can also provide a consistent framework for the design of systems and an evaluation framework in which to assess them. A true test of the generic nature of the platform is best assessed through empirical trials. Trials of the GLP in the higher education, further education and school sectors are described in later sections.

\section{The meaning of learning}

The theoretical background and underpinning for the GLP was provided by Mayes's 'learning framework' (1994). The original Mayes framework (1994)' 1 is illustrated in Figure 1 and can be summarized in the following way:

- Conceptualization: this refers to the student's initial contact with learning material. This could be achieved by attending a lecture or seminar, reading textbooks, watching television, listening to the radio or by using multimedia material on the Web.

- Construction: at this stage students build on the concepts learnt in the conceptualization phase and refine their understanding by working on further tests and 
examples. This could be achieved by carrying out laboratory experiments or working on practical homework, or using multimedia learning materials.

- Dialogue: at this stage students refine their understanding through dialogue and discussion. This could be achieved by participating in tutorials or having informal and sometimes impromptu conversations. On the Web this could be supported by using shared whiteboards, conferencing tools (e.g., audio, video, data conferencing etc.) more complex shared spaces, or discussion groups.

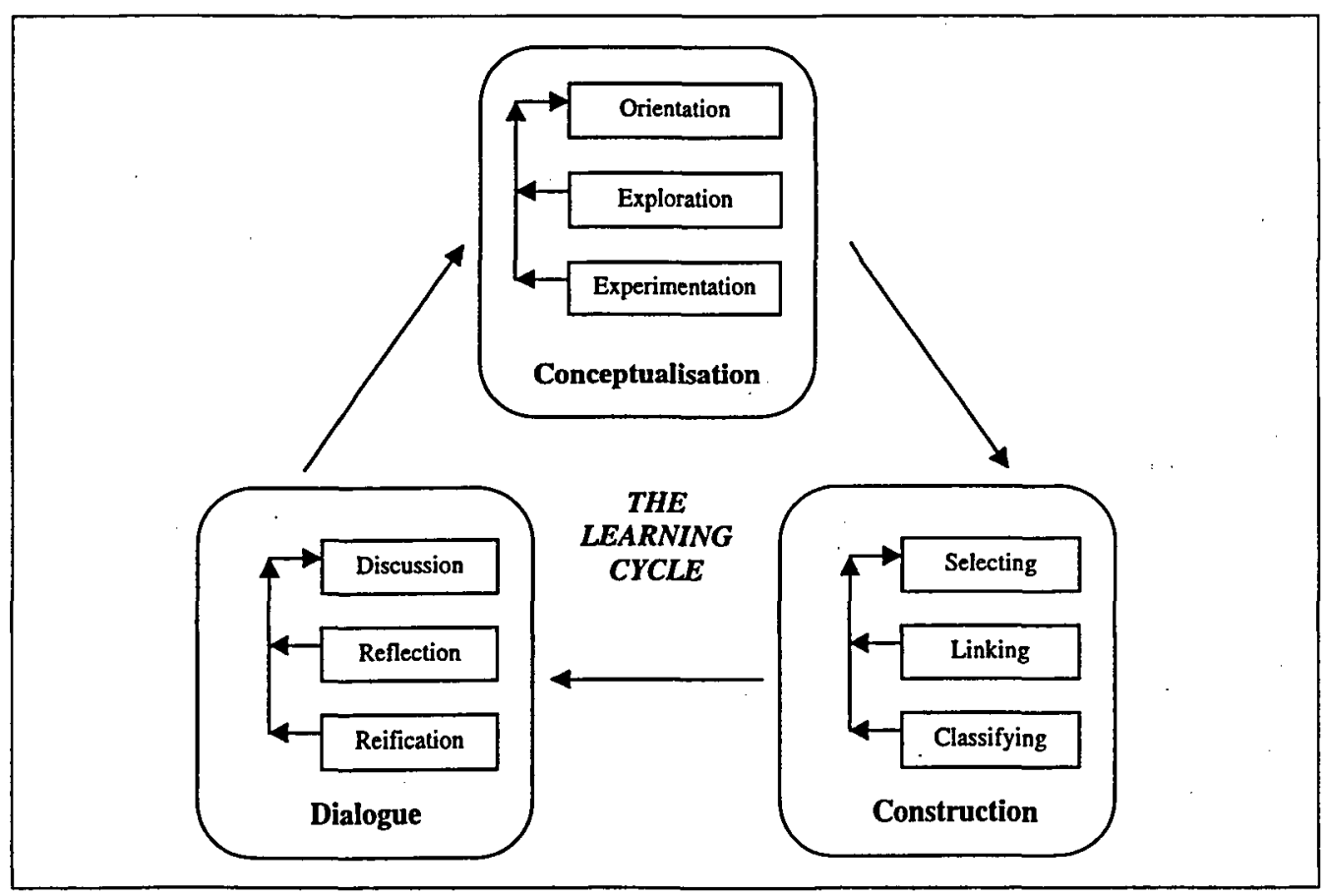

Figure 1: Mayes's conceptual fromework

In general within the sphere of commercial distance learning platforms there has been much greater emphasis on supporting conceptualization, much of it through the development of multimedia Web and CD-ROM content. There tend to be fewer tools to support construction and dialogue as part of an overall educational process. It is for this reason and also the suitability of the constructionist approach to distance learning, i.e. a learner-centred approach, that much of the team's research is aimed at supporting construction and dialogue within this overall educational framework. The key is to identify and support the complete end-to-end 'education process' rather than focus primarily on the end-products of education, e.g. teaching and learning content. The initial requirement therefore for the GLP was that it would support the three-stage Mayes model.

From a commercial perspective the GLP is attractive to BT because it could provide a single service platform which could be used to provide tailored services to different market sectors, i.e. higher and further education (HE/FE), schools, home etc. In this context the 
platform would need to support a much wider range of functions than just those covered in Mayes's model. A service definition for the FE sector, as shown in Figure 2, illustrates this.

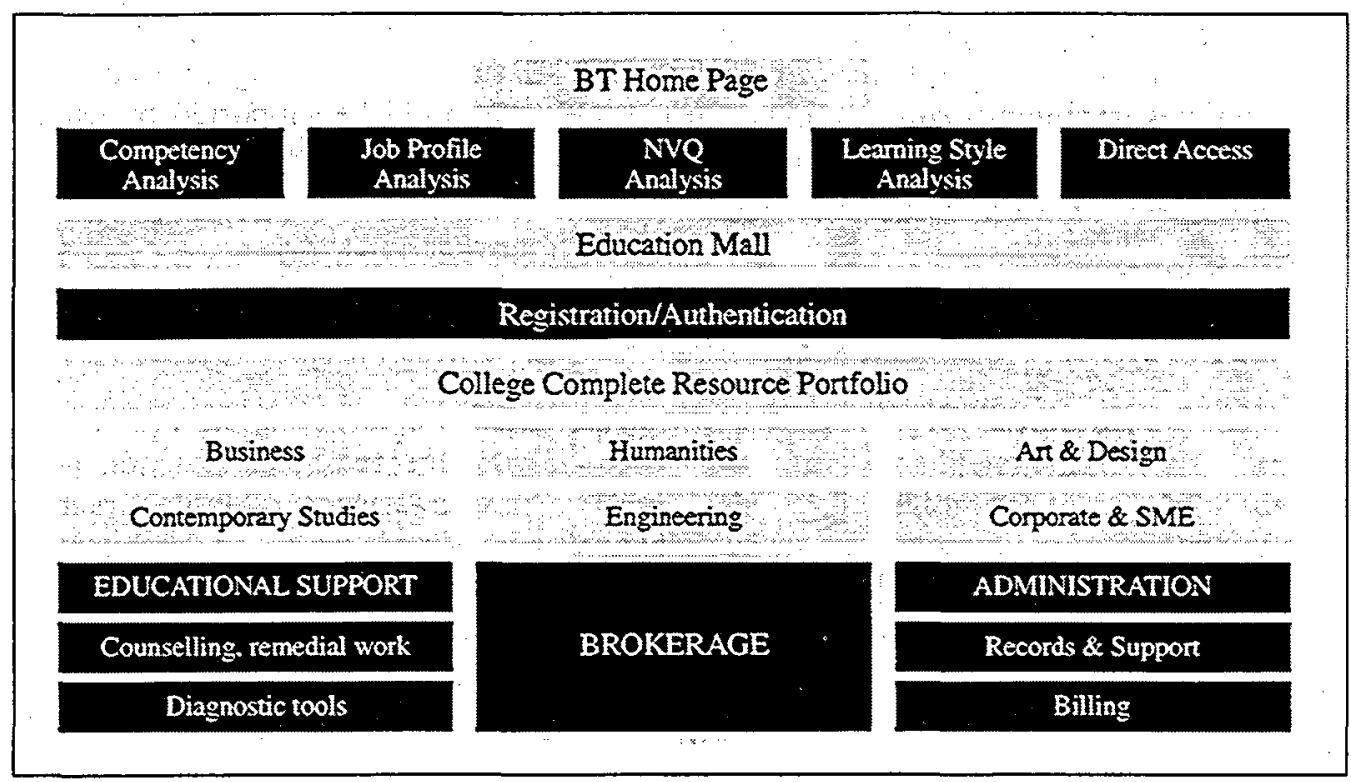

Figure 2: A typical service definition for the FE sector

In this diagram the service definition is made up of five key components:

- Access point: in its simplest form this could be a homepage, but could be more complex if considered as part of a larger range of online educational services.

- Analysis and profiling: tools need to be provided to assist a student or teacher to gain access to the appropriate online resources. For example this can include competency, job profile, or learning style analysis, or it could be based on a defined curriculum, e.g. a National Vocational Qualification (NVQ), or direct access to online resources using advanced search and retrieval tools.

- Administration: this can be the most important component for educational institutions, and will include the user registration and authentication process, tracking, support, billing and content provision.

- Online resources: there will need to be a method for classifying online learning resources and structuring the methods of access. Also in its widest context, online resources can include multimedia content, live and pre-recorded lectures, real people, e.g. tutors, and also resources generated by the students themselves.

- Support functions: these will include a range of support functions such as access to tutors and mentors, counselling and remedial tools.

The BT generic learning platform therefore needed to support not only the Mayes conceptual framework, but also other functions, as defined above, that are necessary to provide a complete online service. 


\section{Development of the RISE platform}

RISE is the instantiation of the GLP that we have developed at BT. Its development has been an iterative process based on a series of trials. These trials were deliberately chosen to stretch the platform and so lead to a better understanding of the issues involved in developing a GLP. This section describes the development of the RISE GLP through research trials.

\section{RISE architecture}

The RISE system had to provide the following core functions:

- Personalized access to online information based on the online group of students, tutors and support staff. Each person would have a personalized information space representing their role within the group and the actual work that they had each completed.

- A virtual classroom or meeting-place which could provide access to good-quality multiparty audio conferencing between participants, and the ability for users to share information and record their conferences.

- Public and private information spaces where students could store their audiorecordings and coursework.

- An online shared diary to enable participants to plan their schedules online.

- Access to a modular set of coursework based on defined stages, modules and checkpoints, plus tools to enable the teaching staff to update and manage the course materials.

- Email and text discussion groups.

- Personalized home pages.

To meet these requirements RISE required three essential features: the integration of an audio conferencing capability with the Web; a dynamic database-driven Web server which could be personalized to each individual user; an online dynamically updating Web Meeting Place. The RISE architecture is illustrated in Figure 3.

RISE is designed around the combination of a data-driven Web server and an audioconferencing server. The Web server is a standard Microsoft Internet Information Server (IIS) on a Windows NT platform. All of the RISE application software is written in Apple WebObjects. WebObjects builds the Web pages dynamically according to the business rules in the RISE application and the underlying data in the Oracle database. In addition to the RISE application there is a separate ConfMan application that manages all conferences and acts as an interface to the Aculab Millennium audio-conferencing platform. The Aculab Millennium is connected to two ISDN-30 lines that will support at any one time up to 60 callers in conferences. Also there is a separate Java server application called REEL. This provides a Web page-sharing capability to users in a conference. Using a simple Java Applet conference participants can push Web pages to each other's browsers. Users of RISE only require a Java-enabled Web browser, e.g. Internet Explorer 3+ or Netscape 3+. No additional client-side software is required. 


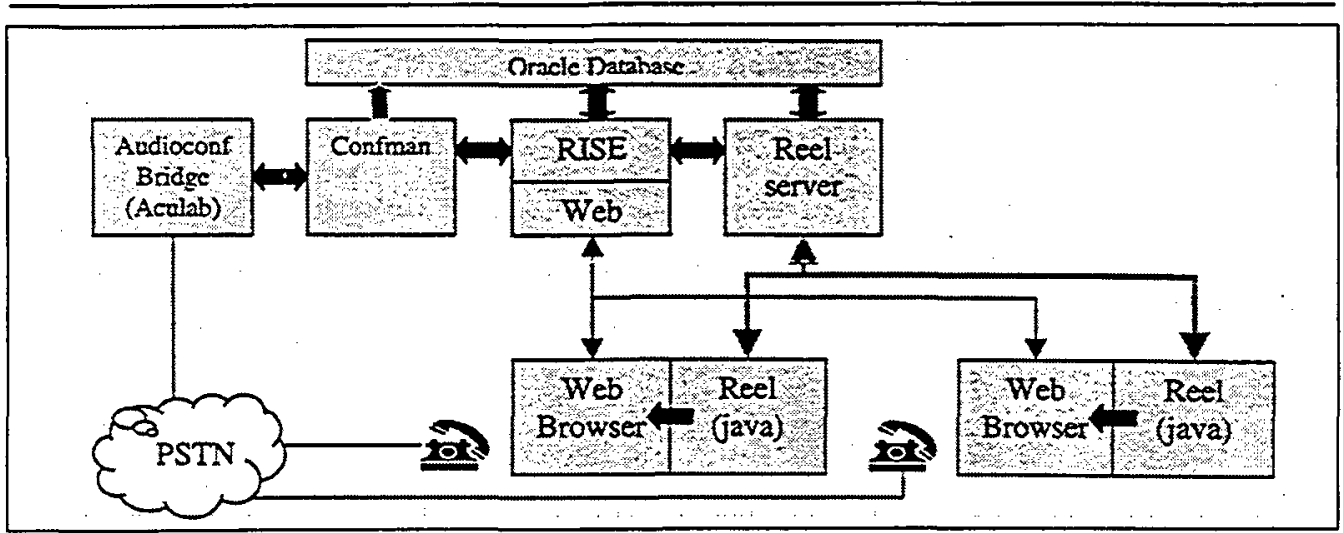

Figure 3: RISE architecture

The key to RISE is the underlying database and the structure of the data schema. Figure 4 shows the data schema used in the Merlin trial. In many ways the success of the GLP will be dependent on the richness and flexibility of this data schema to support a range of different distance learning scenarios. Irrespective of the other technologies used, the data schema defines the distance learning model for the GLP and should be at the core of any new online service.

RISE is built around the ability to create any number of separate closed user groups. Using a number of Web-based online forms a system administrator can create and configure a closed user. For each group, the administrator must configure the group, the roles of users in the group, e.g. tutor, student, administrator, etc. and the layout and functions available to the group. In this way RISE can be used to create a customized system to support a closed user group of individuals. By using the database to build dynamically the online pages for each user it is relatively easy to administer the RISE groups based on the database schema and the customizable options available in RISE. Merlin represents one such closed user group and the Merlin requirements influenced the development of the first version of RISE. However, it was recognized from the start that any GLP must support a large range of different course and learning models, which is why a range of different trials were carried out in order to extend the capabilities of the RISE GLP.

\section{RISE interface and functionality}

The user must first log into RISE with their username and password and the group that they belong to. Once successfully logged in, the user will be presented with two windows, see Figure 5. The larger window contains a toolbar and a workspace area. The toolbar buttons and the 'look and feel' of this window are all dynamically configured for each group. The smaller window is the Web Meeting Place and allows users to see who else is online in their group, enabling them to initiate and take part in audio-conferences with other users. Once in a conference, users can record their conversation, invite other people, and control the privacy of the conference. They can also share Web pages with one another using the REEL Applet, see Figure 6. BT has secured two patents on the RISE system concerning the way the Meeting Place is used to control audio conferences and the use of the REEL application to share Web pages. RISE also provides email and discussion group facilities for all users within the group. 


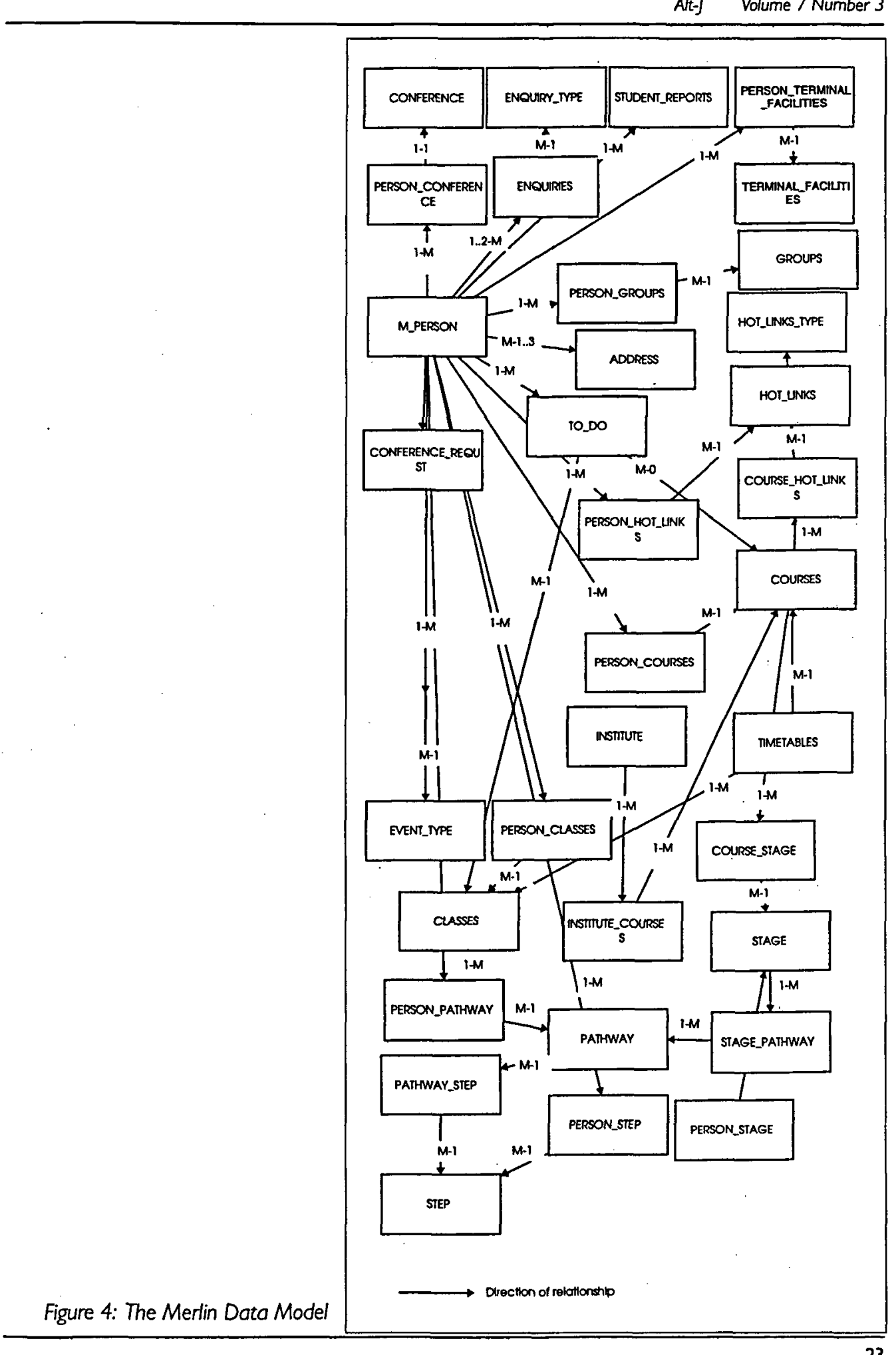




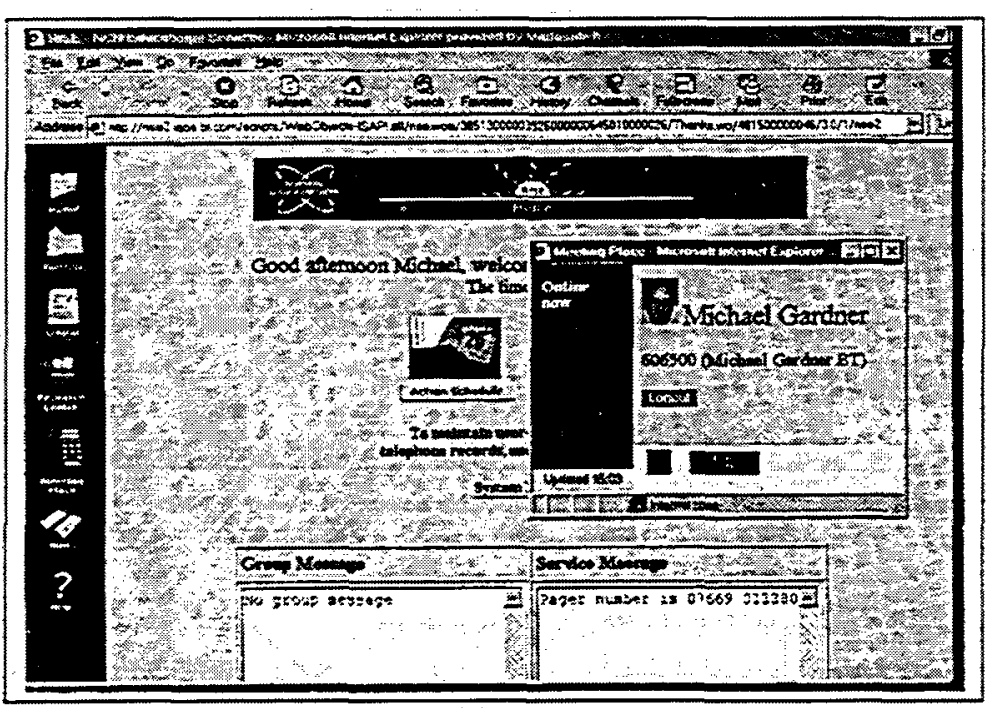

Figure 5: RISE screens

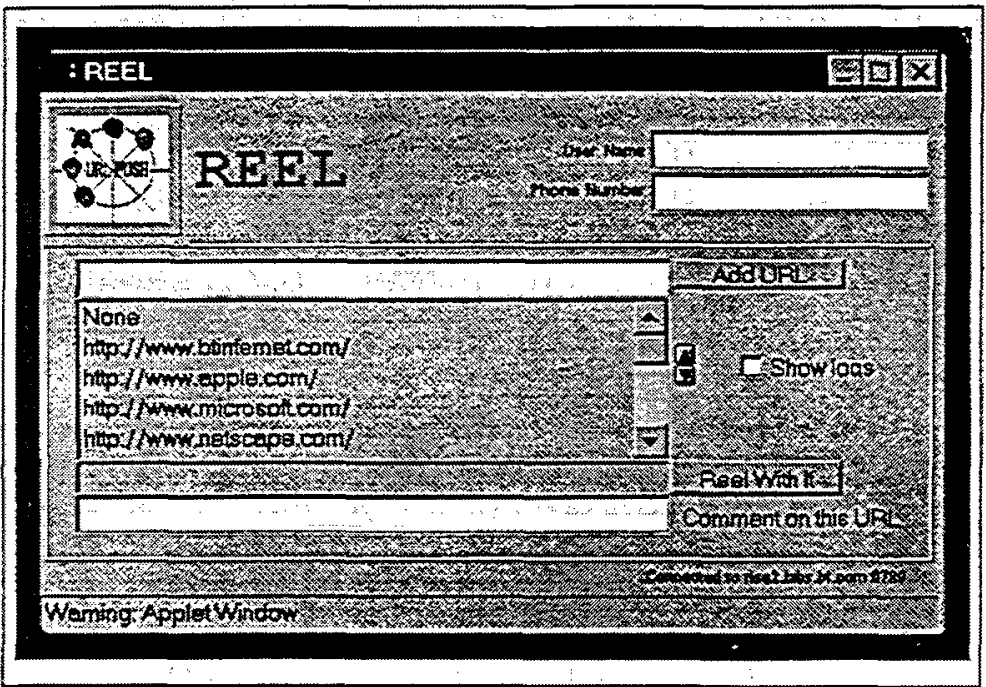

Figure 6: Reel Applet Window

As part of the RISE core functionality each user has access to their own private folder and a shared folder which is available to everyone in the same group. These folders can be used to store any work completed by individuals in the group. Work items can include audiorecordings from RISE conferences or any uploaded computer file. Users can easily upload files from their own PC into these folders for their own use, to share with the tutor, or to share with the whole group. When audio-conferences are recorded the system converts them automatically into RealAudio format. This allows users to stream the audio file back to their PC when they want to listen to it, rather than downloading a large file to their PC. Users can also email the URL of the recording to another person.

In addition to the RISE Meeting Place, the students and teachers also needed a way of scheduling when they would be online, and organizing conferences with one another. It is 
possible to organize conferences formally using asynchronous tools such as email. However, this can be inappropriate if students or teachers want to organize informally their 'online time' to ensure that they will be online when others in their group are also online. To meet this need we developed a shared online diary in RISE, see Figure 7. This allowed users to publicize when they intended to be online. Students could organize their use of the system when others would also be online. This type of informal groupware support is crucial to the success of any distance learning group.

Figure 7: The Merlin Diory

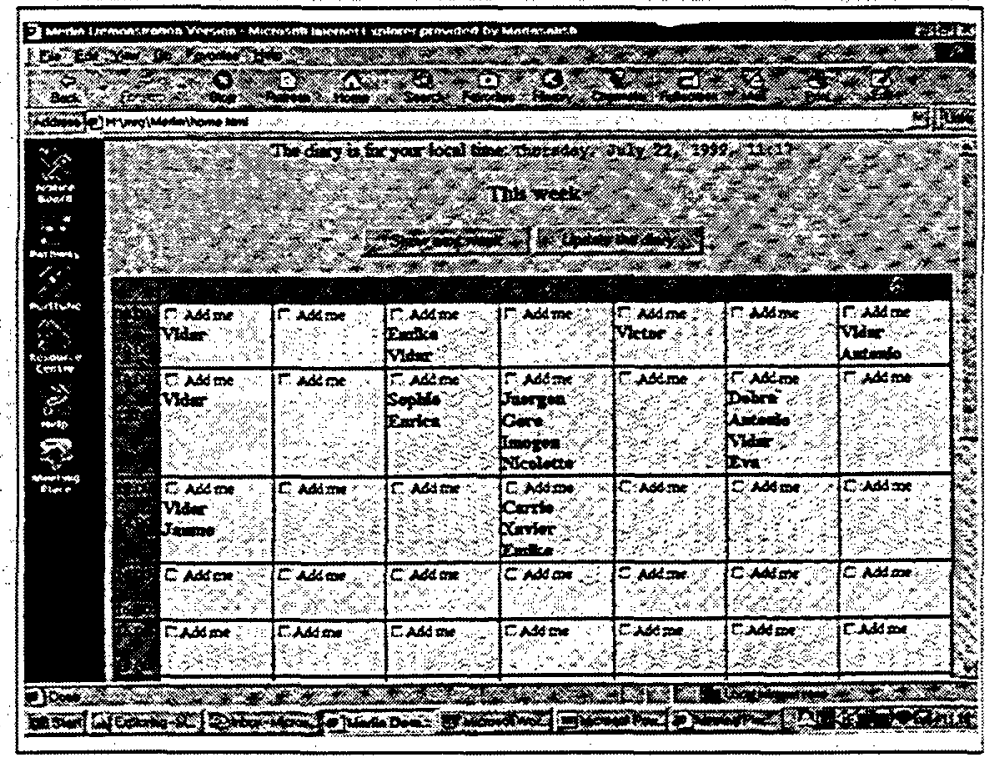

\section{The testing of RISE}

The first use of RISE was in the Merlin trial (Miller, 1996) which ran from October 1996 through to June 1997. The Merlin project was a collaborative project between BT and the University of Hull Language Institute. It was primarily designed around a 16-week English as a Foreign Language (EFL) course delivered online to 40 students world-wide. Merlin was influential as it defined the requirements for the first version of RISE and provided the core features for all subsequent trials. Merlin also set the model for future collaborations, whereby BT acted as a service provider of RISE to an educational organization. Through these trials we learnt a lot about how this relationship could work effectively, how to facilitate the understanding of requirements and how to enable essentially non-technical teaching organizations effectively to deliver courses online via the Web.

Merlin required the following core features:

- Conceptualization: a 16-week modular course with assessment points. The content for the course would be released at set periods under the control of the tutors.

- Construction: students had to be able to practise their English with a peer group and tutors, be able to record their spoken work and share it with others on the course. They had to complete a range of online exercises and save results as well as access standard course materials. 
- Dialogue: students and tutors had to be able to hold both formal and informal meetings using audio conferencing and be able to share information online.

The Merlin trial had distinct requirements in terms of the provision of online courseware to the students. The EFL course was based on a 'pathway' of eight separate stages with checkpoints at the end of each stage, e.g. a formally assessed exercise. Within each stage were a number of separate modules that students could work through to enable them to complete the stage. The key for RISE in this scenario was to enable the course deliverers, Hull University, to manage their courseware and the extrinsic course process. A number of course management tools were developed to meet this end. These tools allowed the Hull team to add dynamically new modules and exercises using bespoke administrator forms. In this way the course material could be released over the 16-week period and the tutors could dynamically update the material based on students' progress and lessons learnt from the students' use of the previous courseware. We also provided the Hull team with a basic Applications Programming Interface (API) which allowed them to develop course material which included online exercises. The API allowed the courseware team to define these exercises in a way that allowed the students to store their answers to the exercises in the Merlin workbook areas, and index these answers against the specific part of the EFL pathway that they came from. In summary, these tools allowed the course providers to have complete control over the online courseware and be able to build assessable course exercises, which could be tracked against each student. In addition to using these tools, any Web-based content could also be linked into the system as courseware or as an online resource.

The Merlin trial had fairly specific requirements for the EFL course based on a strict modular course structure and a rigid curriculum. This type of formal course structure is fairly commonplace. There are many similarities to other school and HE-based curricula. However, particularly in vocational courses there is a need to support a much more flexible course structure, one where the emphasis is more on the learner to assemble evidence of particular competencies that can then be assessed by a tutor, e.g. a student undertaking an NVQ. In 1997, BT ran a second trial using the RISE platform in collaboration with Northern Colleges Network (NCN), a group of FE colleges in the Northumberland region. In addition to testing the core technologies, the key objectives of this trial were to evaluate the usability of a GLP in the FE sector, increase awareness of online learning throughout the FE sector and provide early indications of the acceptability of technology for online learning. In addition to this the team investigated the effects of organizational factors on implementation of online learning (Ward, Tracey and Barker, 1998).

During this trial the BT team worked closely with the NCN tutors and administrators to develop a version of RISE to deliver the NVQ Level II in Information Technology. The key requirement for the RISE platform in this instance was the need to support the NVQ process. To meet this end, a number of enhancements were required. This included an NVQ Schedule Management Tool to allow students to monitor and update their progress against all elements of their NVQ. Tutors could also use the tool to oversee and monitor the students' progress and update the schedule when necessary, e.g. following formal assessment.

Another requirement from the NVQ process was fully to support the collection and assessment of student evidence documents. In Merlin, Personal and Group 'workbooks' 
were provided to allow users to store items such as recordings of conferences and completed coursework. The NVQ process required that the system could more formally support the process of capturing, sharing and assessing evidence material. To meet this need four 'workbook' areas were created:

- an 'audio-evidence' folder, where students could save private recordings from audio conferences;

- a 'meeting records' area, where users could save shared meeting recordings;

- a 'progress review' area, where students could upload documents related to the formal NVQ progress reviews;

- a 'text evidence' folder, which could be used by students to maintain a store of all their documents related to the evidence required.

The access privileges to all of these folders were managed by the RISE system to ensure that students only had access to their own records, and tutors could access all records for all of their students.

So far the RISE platform had only been used to support formal distance learning courses based on a curriculum of task activities and formalized assessment points. The next key question was whether the GLP could be applied to less formalized learning communities, where rather than supporting a set curriculum the GLP needs to support the general communications and learning needs of the community. To investigate this scenario the HomeLearn trial was initiated. This was a collaboration between BT and Apple Computer Inc. and used Wickham Market Primary School in Suffolk as the case study (James, 1999; Tracey, Fowler and Penn, 1999). The aim was to investigate how RISE could be used to support the relationship between home and school. Students from Years 5 and 6 and their families participated in the trial. They were provided with a PC and an Internet connection at home. RISE accounts were created for teachers, students, their families and a school governor. No additional 'content' was created to support this trial as an explicit course was not being run. Instead the team investigated the communication needs of participants. To meet this requirement RISE was extended to include facilities for:

- Tracking and managing students' homework. The teacher could create, assign and monitor homework for the trial students. Students could track their homework and parents could monitor their child's homework and electronically authorize it as being 'seen'.

- Publication and sharing of school's long- and medium-term plans. A tool was developed to allow the teacher to create daily short-term plans and publish them for the trial community.

- Discussion group areas were extended to allow a greater variety of discussion areas. Some were only open to distinct user sub-groups, e.g. children-only or parent-only. This also included project-specific individual and group folder areas, which could be used to support specific activities.

- The RISE conferencing facility was extended to allow invited experts to attend specially organized conferences as part of the students' project work, e.g. a meteorologist in the geography class. 
A further RISE trial was conducted in collaboration with the University of the Highlands and Islands (UHI) in 1999. This used the RISE general purpose group and conferencing facilities to support the delivery of online lectures to a group of students undertaking a software engineering B.Sc. course. Students and lecturer would log into RISE and join together in an audio conference using the RISE Meeting Place. Once in a conference, the lecturer would use the REEL tool to 'push' lecture slides to each student's Web browser whilst giving the lecture as an audio conference. In addition to the UHI trial with RISE, the BT team have developed a number of other tools as part of an Advanced Learning Environments (ALE) project to assist in the capture and synchronization of slides and audio and in archiving and distributing lecture material (see Mudhar and Fowler, 1999).

Our final trial is ongoing and involves working with Suffolk College on a European Social Fund (Adapt) project, COVETS, to develop a 'Televersity' platform for Suffolk. At the heart of the system will be Solstra (http://www.solstra.com/), which will provide all of the user registration and content provision aspects. RISE will be integrated into this system to provide support for the community conferencing and the upload and sharing of individual and group work. The plan is to test this platform through some early trials based on specific courses, but widen this out to address many of the issues raised by the UK Government University for Industry. (UFI) initiative. A particular focus will be on providing education and training to meet the needs of a wide range of different people in the community and laying some of the foundations for the support of lifelong learning.

This section has described the development of the RISE Generic Learning Platform based on a number of trials with different educational contexts. The next section highlights some of the key findings from the evaluation of some of these trials.

\section{Key findings from trials}

\section{System usability}

As with any experimental software the trials encountered some technical problems. Those highlighted in the early trials were overcome and RISE was refined for the following trial. The key problem, identified during the Merlin trial, surrounded the issue of integration. Users were uncomfortable using several applications at the same time and frequently requested a combined user interface from which to launch all applications. To resolve this FirstClass and RISE were integrated into the same browser for the NCN trial. The result was positive feedback from the users. This demonstrated that RISE was a tool with an acceptable level of hidden technology, the user simply logged on and completed a task without identifying that they were using two different applications.

Many of the users expressed a desire to have multi-party video conferencing facilities built in to the RISE platform in addition to the audio conferencing tools already available. Prior research had indicated that users were less interested in 'seeing' one another than they were in actually completing the task at hand, e.g. sharing a Web page, and preferred better quality audio and faster data-sharing facilities than a video image. During the UHI trial BT carried out a comparison of lectures delivered using video conferencing to those delivered over the audio link built in to RISE. The students reported that the quality of the video conferencing was very poor but they still felt that actually being able to see their lecturer was beneficial. However, the lecturer suggested that video conferencing 'didn't add 
a huge amount over audio conferencing'. He was comfortable using RISE to deliver and support his slide material. Despite students' early concerns, at the end of the trial all of the students 'preferred using RISE to the video conferencing' (Tracey, 1999).

It should be noted that students who were already friends or had met their tutor prior to the trials were comfortable conferencing without a video image. Only those who had no previous contact were initially uncomfortable with an audio link alone. Many users commented that making contact with a complete stranger is much harder in a 'virtual' environment than it is in a 'real' classroom. However, as the users familiarized themselves with the system, the course and their peer group, their confidence grew and their need for the visual cues changed. This highlighted the need for a coherent induction process to any course delivered online.

All users used the email actively and found this to be of huge benefit. Students on the UHI trial found this useful as the lecturer was not always online for synchronous communication, but by leaving a text message using FirstClass the students were confident that they would receive a prompt response: 'It was very convenient getting in touch with him [the lecturer] by email.'

On a similar BT research collaboration based in Essex, the School Centred Initial Teacher Training (SCITT) course, tutors actively encouraged remote PGCE students to submit assignments using FirstClass. This was eventually made compulsory and the course manager identified several reasons for this, including the ability to save and file the assignments on the FirstClass server, establish group folders with restricted access for security, and the ability to trace the history of messages. Participants on the NCN trial identified the same benefits.

\section{Teaching staff experiences}

By implementing an online service to deliver and manage a course or series of courses the teaching staff found that their roles changed slightly. They highlighted the issue that they were now expected to be first-line technical support for the student groups regardless of their main subject area. Educational institutions need to review this requirement prior to the implementation of a RISE-type platform to deliver or enhance any course.

However, technical support aside, estimates from the workload of staff, specifically from the Merlin trial, indicated that a tutor could support a larger student group online than in a classroom-based course with a similar input of hours. However, educational institutions must be aware that an increasing number of students increases the level of administrative support required.

Staff also found that they built stronger working relationships with students during the trial. In particular during the Merlin trial the tutor's role was that of a facilitator rather than course leader. Due to this and the increased opportunities for one-to-one online contact the tutor commented that 'there were more opportunities to get to know students'.

The feeling of being better able to address the individual needs of students was common amongst staff in the NCN, Merlin and HomeLearn trials.

\section{Students' feedback}

For all students who took part in the RISE trials the concept of delivering or participating in an online lecture was a new experience. Many of the staff and students expressed 
concerns about what to expect from the system. Even students who considered themselves to be technically competent were wary of how the trial would run. For example one of the students from the UHI trial commented: 'It was a bit of the unknown really. To be quite honest I was a bit unsure about it, I just didn't think it would be as good as somebody standing there in front of a blackboard.'

However, the advantages of a GLP, in particular the flexible working opportunities, soon outweighed the initial concerns. All the students who participated in the UHI trial wanted to use a RISE-type platform again. While this was an experimental platform, the students were undertaking a real course and they achieved 100 per cent pass rate while using RISE.

One of the early questions in all these trials addressed the issue of student-tutor interaction. How would it be affected by the technology? The majority of HE/FE students considered the amount of support or contact with their tutor or lecturer compared favourably with that from classroom teachers. For Merlin students the spoken contact with the tutor was more enjoyable and beneficial than in classrooms. For the students participating in the NCN trial the ability to send and receive emails and book online meetings with their tutor was essential and they felt they received better attention than if they were ir a group tutorial or meeting. NVQs usually involve less contact with a tutor and according to the students the introduction of RISE had improved this level of interaction.

\section{Learning gains}

The objectives for the students during the NCN trial included using RISE to download courseware related to their NVQ, begin the exercises and start to gather evidence towards their NVQ. Three-quarters of the students had downloaded courseware and one-third had reached the stage of submitting evidence. Over 80 per cent of the student group felt that they had benefit in terms of ICT skills, knowledge of GLPs and general confidence levels using a GLP (Ward, Tracey and Barker, 1998). Of the RISE trials this was the only instance where students participated voluntarily and the trial was in addition to their work and study commitments. All other trials were delivering 'live' courses.

The use of RISE in the community environment during the HomeLearn trial increased the students' access to resources. For example, an audio conference with the author of their current English textbook was arranged after school as a homework assignment. The children had the opportunity to ask the author specific questions and engage in spontaneous debate following the answers. This and other similar activities not only enabled the students to complete their homework but gave them the opportunity to develop ICT skills, presentation, interpersonal and communication skills and research skills. They could achieve all this from their own homes with the support of either friends or family as well as receive the support and guidance from teaching staff in the formal classroom environment.

\section{Conclusions}

A GLP works very well as the sole learning environment in a situation whereby the student is self-motivated and does not consider the teaching staff to be the sole or principal learning resource, e.g. Merlin or NCN trials. In this case students manage the majority of their learning experience. As a communication tool used to bring together communities 
that ordinarily would have less contact, e.g. the school and parents in the HomeLearn trial, RISE is very successful but would not replace the formal classroom-based environment that young children require. It can provide an excellent gateway to exploring a global resource bank, via the Web and email, to supplement and support the National Curriculum, but should not be considered as a replacement for the teacher during compulsory years.

\section{The future}

This paper has reviewed the work done in BT to develop a GLP through the development of RISE. BT is now developing a commercial platform that delivers many of the features that were previously provided by RISE, and work is continuing, e.g. COVETS, to extend features and coverage of the new commercial platform.

With the growing maturity of this area, international standards will have a much larger part to play in ensuring that there is consistency in all aspects of distance learning. In particular, the Instructional Management Systems (IMS) Project (1999), an Educom NLII initiative, is developing a specification and software for managing online learning resources, where learning resources can include people, educational service companies, content, tools and activities. With the rapid increase in the availability of online educational content a common methodology and language for describing online learning resources is required. Content can often be described in many different ways. These descriptive categories are meta-data about the object. Online materials need a similar system of meta-data.

Standards are also emerging for the design of the generic learning platform itself, notably the IEEE Learning Technology Systems Architecture (LTSA) as described by Farance and Tonkel (1998). This Architecture Specification describes the high-level system design and the components of the LTSA. The LTSA specification covers a wide range of systems, including learning technology, computer-based training, electronic performance support systems, computer-assisted instruction, intelligent tutoring, education and training technology, and meta-data. The LTSA specification attempts to provide a framework for understanding existing and future systems, and promotes interoperability and portability by identifying critical system interfaces such that any system can comply with the LTSA.

BT is continuing to carry out research into GLPs, in particular:

- investigating new types of content to support collaboration and construction;

- addressing psychological issues of distance learning, e.g. how to engage learners, and new literacies required by learners;

- understanding the needs of complex learning communities and mapping these through to the design of online support systems.

In BT, RISE has provided us with a rich understanding of how distance learning can be effectively supported and through a variety of trials has shown how a single GLP can support a range of different learning communities. 


\section{Note}

1 The framework has been further developed - see Fowler and Mayes in this edition for more details.

\section{References}

Fowler, C. J. H. and Mayes, J. T. (1997), 'Applying telepresence to education', BT Technology Journal, 14, 4, 98-110.

Farance, F. and Tonkel, J. (May, 1998), 'Learning Technology Systems Architecture (LTSA) Specification', Version 4.00, A Base Document for IEEE 1484.1, http://www.edutool.com/ltsa.

James, G. (April 1999), 'Inviting the world into your school', Interactive Journal, 27-9.

IMS Meta-data project (October, 1999), IMS Meta-data Specification, http://www.imsproject.org/metadata/.

Mason, R. (April 1998), 'Models of OnLine courses', Networked Lifelong Learning (NLL), '98 Conference Proceedings, 1.72-1.79.

Mayes, J. T. (1994), 'Learning through telematics', A Report Commissioned by BT Report, (C) BT Telecommunications Plc.

Miller, C. (1996), 'Initial user evaluation of Merlin', BT Internal Report, (C) BT Telecommunications Plc.

Mudhar, P. and Fowler, C. J. H. (1999), 'The advanced learning environment (ALE) demonstrator: from theory to design', Computer Aided Learning (CAL) '99 Abstract Book, 49.

Smythe, P. and Gardner, M. (1997), 'The RISE Platform: supporting social interaction for on-line education', CHI 97 Electronic Publications: Late-Breaking/Short Demonstrations.

Tracey, K. (June 1999), 'UHI evaluation report', BT Internal Report, (C) BT Telecommunications Plc.

Tracey, K., Fowler, C. J. H. and Penn, C. (January 1997), 'Developing an infrastructure to support communities of learning', BT Technology Journal, 17, 1, 98-110.

Ward, H., Tracey, K. and Barker, M. (April 1998), 'Developing a virtual college', Networked Lifelong Learning, '98 Conference Proceedings, 4.43-4.51. 